\title{
Analysis of Pull-in Characteristics of Double-clamped Nanobeam Incorporating Casimir and van der Waals Effects
}

\author{
Cheng-Chi Wang, ${ }^{1}$ Chih-Jer Lin, ${ }^{2}$ Chin-Chia Liu, ${ }^{3 *}$ and Chia-Chiang $\mathrm{Hsu}^{3}$ \\ ${ }^{1} \mathrm{Ph} . \mathrm{D}$. Program, Graduate Institute of Precision Manufacturing, \\ National Chin-Yi University of Technology, Taichung County 411, Taiwan, R.O.C. \\ ${ }^{2}$ Graduate Institute of Automation Technology, National Taipei University of Technology, \\ 1, Sec. 3, Zhongsiao E. Road, Taipei 10608, Taiwan, R.O.C. \\ ${ }^{3}$ Department of Industrial Education and Technology, National Changhua University of Education, \\ Bao-Shan Campus, Number 2, Shi-Da Road, Changhua 500, Taiwan, R.O.C.
}

(Received March 28, 2018; accepted September 7, 2018)

Keywords: double-clamped nanobeam, NEMS, pull-in voltage, Casimir force, van der Waals (vdW) force

A hybrid numerical scheme comprising the differential transformation method (DTM) and the finite difference (FD) approximation approach is used to analyze the complex nonlinear pull-in behavior of an electrostatically actuated double-clamped nanobeam subject to Casimir and van der Waals force effects and an axial residual stress. It is shown that, for an initial gap size of $50 \mathrm{~nm}$, the pull-in voltage predicted by the hybrid numerical scheme deviates from that predicted by the universal pull-in formula by just $0.2 \%$. In addition, the results show that the Casimir and van der Waals forces both have a significant effect on the steady and dynamic deflection behaviors of the beam as a function of applied voltage. Finally, the minimum allowable gap without the applied voltage of double-clamped nanobeams can be determined.

\section{Introduction}

Nano-electromechanical system (NEMS) devices have found widespread use throughout the industry for such applications as nanoswitches, ${ }^{(1)}$ nanotweezers, ${ }^{(2)}$ and ultrasensitive sensors. ${ }^{(3)}$ NEMS devices commonly incorporate beam-type electrostatic actuators consisting of two conductive electrodes (one fixed and the other movable) separated by a dielectric spacer. When a voltage difference is applied across the two electrodes, the upper electrode deflects in the downward direction as a result of electrostatic forces. At a certain critical voltage, defined as the pull-in voltage, the electrostatic force exceeds the elastic restoring force, and the electrode collapses onto the lower fixed electrode. The pull-in behavior of MEMS actuators has attracted significant interest in recent decades. Hung and Senturia ${ }^{(4)}$ utilized the leveraged bending and strain stiffening methods to extend the travel range of analog-tuned electrostatic actuators prior to pull-in. Liu and Wang ${ }^{(5)}$ evaluated the effects of squeeze-film damping on the pullin voltage of clamped-clamped microbeams. Sadeghian et al. ${ }^{(6)}$ applied the generalized

"Corresponding author: e-mail: ccliu@cc.ncue.edu.tw https://doi.org/10.18494/SAM.2018.2081 
differential quadrature method (DQM) to the investigation of the pull-in phenomena of MEMSbased microswitches. Kuang and Chen ${ }^{(7)}$ used the Adomian decomposition method (ADM) to examine the nonlinear pull-in behavior of microactuators.

At the nanoscale, defined as characteristic dimensions of $100 \mathrm{~nm}$ or less, various new and exciting physical phenomena occur, causing many properties to vary significantly from those observed at a larger scale. Thus, in analyzing and designing NEMS devices, the effects of intermolecular forces such as the van der Waals force ${ }^{(8)}$ and Casimir force ${ }^{(9)}$ must be taken into account. Both forces vary inversely with the separation distance between the two electrodes, and hence NEMS devices exhibit an inherently nonlinear behavior. For separation distances less than $20 \mathrm{~nm}$, the attraction force is dominated by the van der Waals force. However, for separation distances greater than $20 \mathrm{~nm}$, the force between the two surfaces is governed by the Casimir effect. ${ }^{(10)}$ Ramezani et al. ${ }^{(11)}$ presented a distributed parameter model for the pullin instability analysis of electrically actuated nanocantilevers subject to van der Waals and Casimir force effects. Rotkin ${ }^{(12)}$ presented a general analytical model for describing the effect of the van der Waals force on the pull-in voltage and pull-in gap of NEMS systems. Jia et al. ${ }^{(13)}$ used the DQM method to study the free vibration behavior of nano-/microbeams under different boundary conditions with the combined effects of electrostatic force, axial residual stress, nonlinear midplane stretching, and Casimir force.

The differential transformation method (DTM) is a numerical technique based on Taylor series expansion for the solution of differential equations in the form of polynomials. ${ }^{(14)}$ DTM requires neither linearization nor perturbation and provides an effective, straightforward, and accurate means of solving a wide range of linear and nonlinear problems. ${ }^{(15)}$ Although originally proposed for the solution of problems in the electrical circuit domain, DTM has been extended to a wide variety of problems in the engineering field in recent years. For example, Liu and coworkers ${ }^{(16,17)}$ used DTM to investigate the problem of entropy generation within a mixed convection flow with viscous dissipation effects. The same group ${ }^{(18-20)}$ used a hybrid method combining the DTM approach and the finite difference (FD) approximation method to analyze the nonlinear dynamic behavior of micro-electromechanical circular plates subject to squeeze-film damping effects.

In this study, the hybrid numerical scheme is used to examine the nonlinear deflection behavior of a double-clamped nanobeam. The validity of the proposed method is demonstrated by comparing the predicted pull-in voltages for various initial gap sizes with the results obtained using the universal pull-in formula presented in Ref. 8. The validated method is then used to explore the effects of the van der Waals and Casimir forces on the pull-in behavior of the clamped nanobeam given various values of the axial residual stress. In general, the results show that the hybrid numerical scheme provides an accurate method of analyzing the nonlinear pull-in behavior of double-clamped nanobeams and similar NEMS-based structures.

\section{Double-clamped Nanoactuator Model}

The analysis performed in this study considers the double-clamped nanobeam actuator shown in Fig. 1. As shown, the upper beam is actuated by a driving voltage $V$. At a certain 


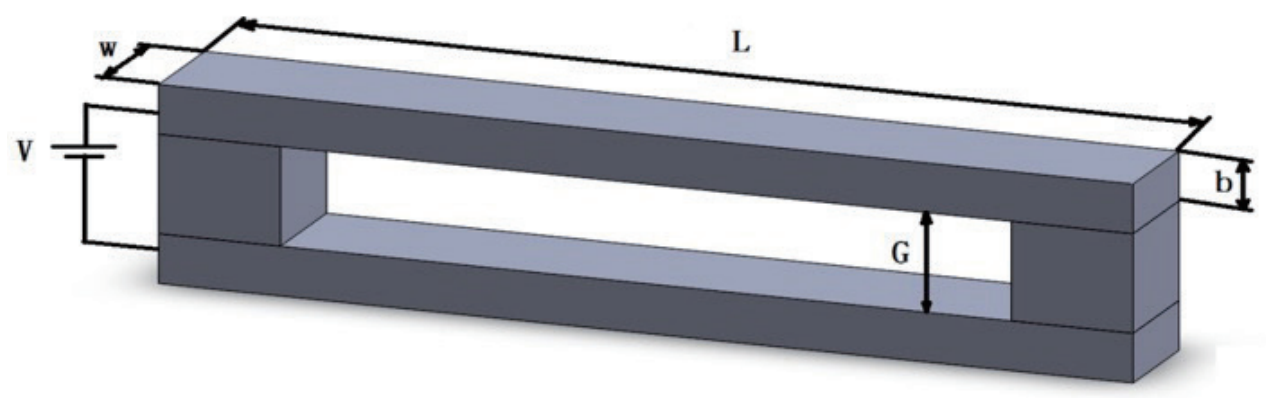

Fig. 1. Schematic illustration of double-clamped nanobeam.

critical voltage, the magnitude of the electrostatic force between the upper and lower beams exceeds the restoring force within the upper beam, and thus pull-in occurs. The doubleclamped nanobeam actuator is composed of length $L$ with a cross section of thickness $b$ and width $w$. The initial gap between the movable beam and the fixed electrodes is denoted by $G$.

The governing equation for a double-clamped nanobeam subject to electrostatic actuation, intermolecular forces, and von Kármán nonlinear midplane stretching has the form ${ }^{(21)}$

$$
\tilde{E} I \frac{\partial^{4} h}{\partial x^{4}}+\rho A \frac{\partial^{2} h}{\partial t^{2}}-\left[\frac{\tilde{E} A}{2 L} \int_{0}^{L}\left(\frac{\partial h}{\partial x}\right)^{2} d x+T_{r}\right] \frac{\partial^{2} h}{\partial x^{2}}=F_{\text {elec }}+F_{n}, n=3,4
$$

where $h$ is the transverse (i.e., vertical) displacement of the beam at position $x$ and time $t$ [i.e., $h=h(x, t)]$, and $\tilde{E}$ is the effective modulus of the nanobeam material. For a wide nanobeam (i.e., $w \geq 5 b), \tilde{E}$ is equivalent to the plate modulus $E /\left(1-v^{2}\right)$, where $i$ is the Poisson ratio. By contrast, for a narrow beam, the effective modulus $\tilde{E}$ is simply equal to the Young's modulus $E$. In addition, $I$ in Eq. (1) is the moment of inertia of the nanobeam and is given by $I=w b^{3} / 12$, $\rho$ is the material density, and $A=w \times b$ is the cross-sectional area. $T_{r}$ is the axial load acting on the beam and is given by $T_{r}=\tilde{\sigma} w b$, where $\tilde{\sigma}$ is the residual stress and has the form $\tilde{\sigma}=\sigma(1-v)$, in which $\sigma$ is the biaxial residual stress. Finally, $F_{\text {elec }}$ is the electrostatic force, and the index $n$ has values of $3\left(F_{3}\right)$ for the van der Waals force interaction and $4\left(F_{4}\right)$ for the Casimir force interaction.

The first-order fringing field correction of the electrostatic force per unit length of the beam is given by ${ }^{(20)}$

$$
F_{\text {elec }}=\frac{\varepsilon_{0} w V^{2}}{2(G-h)^{2}}\left(1+0.65 \frac{G-h}{w}\right)
$$

where $\varepsilon_{0}$ is the the permittivity of free space. In addition, the Casimir force per unit length of the beam is expressed as ${ }^{(21)}$ 


$$
F_{4}=\frac{\pi^{2} \hbar c w}{240(G-h)^{4}}
$$

where $\hbar$ is Planck's constant $\left(1.055 \times 10^{-34} \mathrm{~J} \cdot \mathrm{s}\right)$ divided by $2 \pi$ and $c$ is the speed of light $\left(2.998 \times 10^{8} \mathrm{~m} / \mathrm{s}\right)$. The van der Waals force per unit length of the beam is derived as ${ }^{(21)}$

$$
F_{3}=\frac{A_{H} w}{6 \pi(G-h)^{3}}
$$

where $A_{H}$ is the Hamaker constant.

In analyzing the nonlinear deflection behavior of the double-clamped nanobeam, the boundary conditions are set as

$$
\begin{array}{ll}
h(x, t)=\frac{\partial h(x, t)}{\partial x}=0 & \text { at } x=0, \\
h(x, t)=\frac{\partial h(x, t)}{\partial x}=0 & \text { at } x=L .
\end{array}
$$

Finally, the initial condition of the double-clamped nanobeam is given as

$$
h(x, 0)=\frac{\partial h(x, 0)}{\partial t}=0 .
$$

For analytical convenience, let the transverse displacement of the nanobeam, $h$, be normalized with respect to the initial gap between the electrodes, the axial length position $x$ be normalized with respect to the nanobeam length, and the time $t$ be normalized with respect to a time constant $\bar{T}$, where $\bar{T}$ is defined as $\bar{T}=\sqrt{\rho A L^{4} / \tilde{E} I}$. That is,

$$
\bar{h}=\frac{h}{G}, \bar{x}=\frac{x}{L}, \bar{t}=\frac{t}{\bar{T}}, \bar{w}=\frac{w}{G} .
$$

In addition, let the following parameters also be defined as

$$
\begin{gathered}
\bar{T}_{r}=\frac{T_{r} L^{2}}{\tilde{E} I}, A_{1}=\frac{\varepsilon_{0} \bar{w} V^{2} L^{4}}{2 \tilde{E} I G^{2}}, A_{2}=0.65\left(\frac{G}{w}\right) A_{1}, \bar{F}_{3}=\frac{A_{H} \bar{w} L^{4}}{6 \pi(\tilde{E} I) G^{3}}, \\
\bar{F}_{4}=\frac{\pi^{2} \hbar c \bar{w} L^{4}}{240(\tilde{E} I) G^{5}}, \alpha=6\left(\frac{G}{b}\right)^{2},
\end{gathered}
$$

where $\bar{T}_{r}, A_{1}, F_{3}, \bar{F}_{4}$ and $\alpha$ are the dimensionless parameters of axial force, electrostatic force, van der Waals force, Casimir force, and gap size, respectively. By substituting Eq. (7) into Eqs. (1), (5), and (6), the dimensionless nonlinear governing equation of the double-clamped nanobeam is obtained as 


$$
\frac{\partial^{4} \bar{h}}{\partial \bar{x}^{4}}+\frac{\partial^{2} \bar{h}}{\partial \bar{x}^{2}}-\left(\bar{T}_{r}+\alpha \int_{0}^{1}\left(\frac{\partial \bar{h}}{\partial \bar{x}}\right)^{2} d \bar{x}\right) \frac{\partial^{2} \bar{h}}{\partial \bar{x}^{2}}=\frac{A_{1}}{(1-\bar{h})^{2}}+\frac{A_{2}}{1-\bar{h}}+\frac{\bar{F}_{n}}{(1-\bar{h})^{n}}, n=3,4
$$

The corresponding boundary conditions are given by

$$
\begin{array}{ll}
\bar{h}(\bar{x}, \bar{t})=\frac{\partial \bar{h}(\bar{x}, \bar{t})}{\partial \bar{x}}=0 & \text { at } \bar{x}=0, \\
\bar{h}(\bar{x}, \bar{t})=\frac{\partial \bar{h}(\bar{x}, \bar{t})}{\partial \bar{x}}=0 & \text { at } \bar{x}=1 .
\end{array}
$$

Finally, the initial condition is defined as

$$
\bar{h}(\bar{x}, 0)=\frac{\partial \bar{h}(\bar{x}, 0)}{\partial \bar{t}}=0 .
$$

\section{Solution of Dimensionless Nonlinear Governing Equation}

In this study, the dimensionless nonlinear governing equation of the double-clamped nanobeam given in Eq. (9) was solved using the hybrid DTM/FD numerical scheme. The solution procedure commenced by discretizing the equation of motion with respect to the time domain $t$ using the DTM method, ${ }^{(17,19)}$ i.e.,

$$
\begin{aligned}
& T\left[\frac{\partial^{4} \bar{h}}{\partial \bar{x}^{4}}\right]=\frac{d^{4} \bar{U}(\bar{x}, k)}{d \bar{x}^{4}}, \\
& T\left[\frac{\partial^{2} \bar{h}}{\partial \bar{t}^{2}}\right]=\frac{(k+1)(k+2)}{H_{t}^{2}} \bar{U}(\bar{x}, k+2) \\
& T\left[A_{1}\right]=A_{1} \delta(k) \\
& T\left[2 A_{1} \bar{h}\right]=2 A_{1} \bar{U}(\bar{x}, k) \\
& T\left[3 A_{1} \bar{h}^{2}\right]=3 A_{1}(\bar{U}(\bar{x}, k) \otimes \bar{U}(\bar{x}, k))=3 A_{1}\left(\sum_{\ell=0}^{k} \bar{U}(\bar{x}, l) \bar{U}(\bar{x}, k-l)\right), \\
& T\left[4 A_{1} \bar{h}^{3}\right]=4 A_{1}\left(\sum_{l=1}^{k}[(3+1) l-k] \bar{U}(\bar{x}, l) \bar{U}^{3}(\bar{x}, k-l)\right),
\end{aligned}
$$

where $\bar{U}(\bar{x}, k)$ is the spectrum of $\bar{h}(\bar{r}, \bar{t}), k$ and $l$ are transformation parameters, and $H_{t}$ is the time interval. Having discretized the governing equation, the associated boundary conditions, and the initial condition, the transverse displacement of the beam was discretized spatially in the length direction using the FD approximation method based on fourth- and second-order accurate central difference formulae. ${ }^{(17,19,20)}$ 


\section{Results and Discussion}

\subsection{Comparison and validation}

The validity of the proposed hybrid numerical scheme was demonstrated by comparing the results obtained for the pull-in voltage of the double-clamped nanobeam with those presented in Ref. 8 based on the universal pull-in formula. In performing the analysis, the material and geometry properties of the nanobeam were assigned the values given in Table 1. Furthermore, an assumption was made that the nanobeam was actuated by a DC voltage. As shown in Table 2, for an initial gap size of $15 \mathrm{~nm}$, the pull-in voltage predicted by the hybrid numerical scheme $(0.44 \mathrm{~V})$ was identical to that predicted by the universal pull-in formula $(0.44 \mathrm{~V})$. Furthermore, for a gap size of $50 \mathrm{~nm}$, the pull-in voltage predicted by the hybrid numerical scheme $(5.1 \mathrm{~V})$ deviated from that predicted by the universal pull-in formula $(5.11 \mathrm{~V})$ by just $0.2 \%$. Thus, the validity of the proposed hybrid numerical scheme is confirmed.

\subsection{Effects of Casimir and van der Waals forces on nanobeam dynamic behavior}

Figure 2 shows the effect of the Casimir force on the variation of the nanobeam center-point displacement over time for various actuating voltages. The material and geometry parameters considered in the present analyses are summarized in Table 1. Note that the initial gap size is set as $50 \mathrm{~nm}$ and the beam is assumed to have no residual stress. In the absence of a Casimir force effect, the pull-in voltage is $5.14 \mathrm{~V}$. However, the pull-in voltage decreases to $5.12 \mathrm{~V}$ when the Casimir force is taken into account.

Table 1

Assumed parameter values of double-clamped nanobeam.

\begin{tabular}{lcc}
\hline Symbol & Parameters & Value/unit \\
\hline$E$ & Young's modulus & $80(\mathrm{GPa})$ \\
$v$ & Poisson's ratio & 0.42 \\
$\rho$ & Density & $19300\left(\mathrm{~kg} / \mathrm{m}^{3}\right)$ \\
$\varepsilon_{0}$ & Permittivity of free space & $8.85 \times 10^{-12}(\mathrm{~F} / \mathrm{m})$ \\
$w$ & Width of beam & $1(\mu \mathrm{m})$ \\
$b$ & Thickness of beam & $100(\mathrm{~nm})$ \\
$L$ & Length of beam & $5(\mu \mathrm{m})$ \\
$A_{H}$ & Hamaker constant & $44 \times 10^{-20}(\mathrm{~J})$ \\
\hline
\end{tabular}

Table 2

Comparison of results obtained for pull-in voltage for different initial gap sizes.

\begin{tabular}{lccc}
\hline & \multicolumn{2}{c}{ Pull-in voltage $(\mathrm{V})$} & Error \\
\cline { 2 - 4 } Initial gap $(\mathrm{nm})$ & $\begin{array}{c}{ }^{(1)} \text { Hybrid numerical } \\
\text { scheme }\end{array}$ & $\begin{array}{c}{ }^{(2)} \text { Universal pull-in } \\
\text { formula }\end{array}$ & $\Delta e(\%)$ \\
\hline 15 & 0.44 & 0.44 & 0.00 \\
\hline 50 & 5.10 & 5.11 & 0.20 \\
\hline$\Delta e(\%)=|(2)-(1)|(2)$ & & &
\end{tabular}




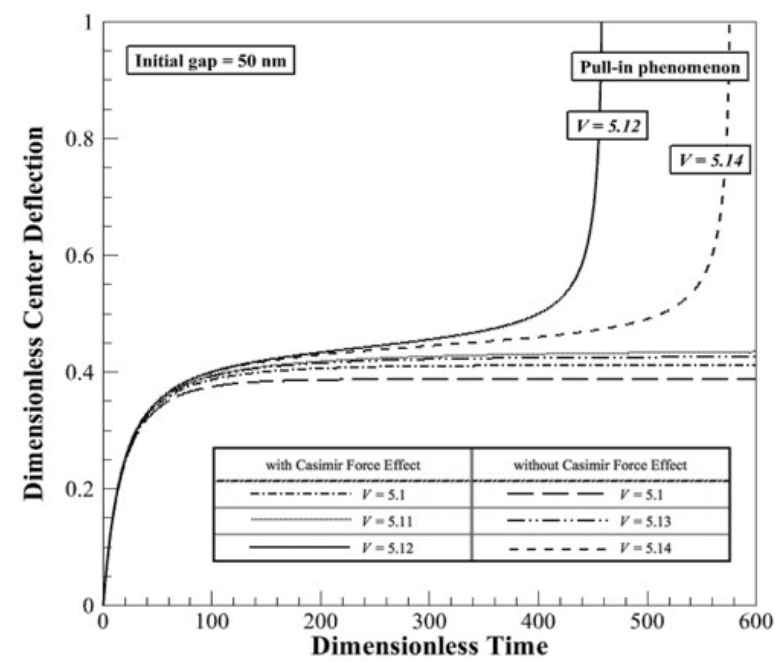

Fig. 2. Variation of dimensionless center-point deflection over time with and without Casimir force effect.

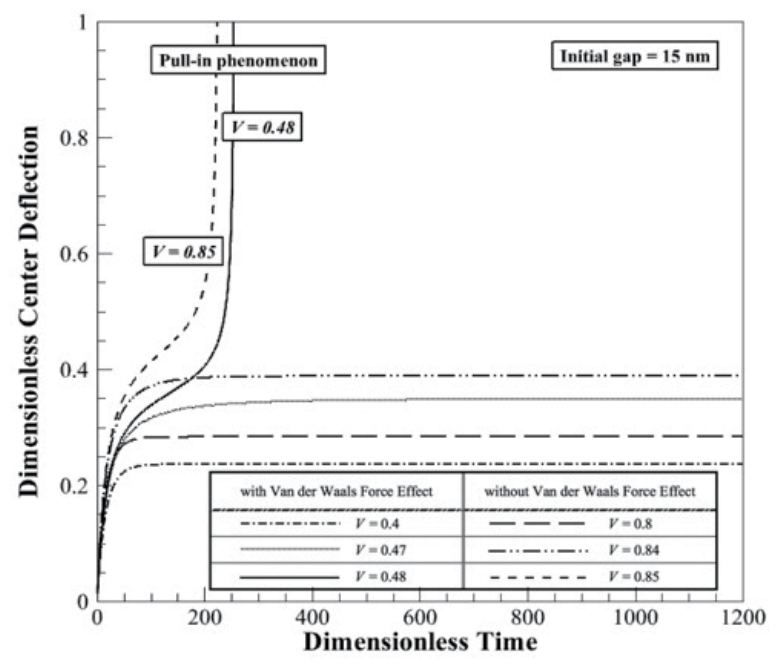

Fig. 3. Variation of dimensionless center-point deflection over time with and without van der Waals force effect.

Figure 3 shows the variation of the nanobeam center-point deflection over time with and without the van der Waals force effect for actuating voltages in the range of $0.4-0.85 \mathrm{~V}$. Note that the effects of residual stress are once again ignored. As for the Casimir force, the pull-in voltage decreases when the van der Waals force is taken into account [i.e., from 0.85 (without van der Waals force) to $0.48 \mathrm{~V}$ (with van der Waals force)]. Overall, the results presented in Figs. 2 and 3 show that the Casimir and van der Waals forces both play a key role in determining the deflection behavior of the double-clamped nanobeam.

Figure 4 shows the variation of the nanobeam center-point deflection with the actuating voltage as a function of the residual stress and initial gap size. Note that $h \geq 1$ is used for the pull-in condition in this study. The pull-in instability can be observed when the slope of the deflection-voltage graph reaches infinity. Note that the effects of Casimir and van der Waals forces are both taken into account. It is seen that, for a constant residual stress, the pullin voltage increases as the initial gap size increases owing to the corresponding reduction in attractive electrostatic force. Furthermore, for a constant initial gap size, the pull-in voltage increases as the residual stress changes from a negative (i.e., compressive) value to a positive (i.e., tensile) value. For example, given an initial gap size of $50 \mathrm{~nm}$, the pull-in voltage increases from 5.0 to $5.23 \mathrm{~V}$ as the residual stress changes from -10 to $10 \mathrm{MPa}$.

Owing to the complexity of the interactions between the electrostatic coupling effect and the nonlinear electrostatic force, the size effect on the nanostructures of dynamic behavior is investigated. Figure 5 shows the variation of the dimensionless center-point deflection with the gap size parameter as a function of applied voltage. Note that the beam is assumed to have no residual stress, and the other properties are presented in Table 1. As expected, the amplitude of the nanobeam deflection decreases with increasing gap size owing to a corresponding increase in initial gap size. Moreover, for a constant gap size parameter, the dimensionless center-point deflection increases with increasing voltage. 


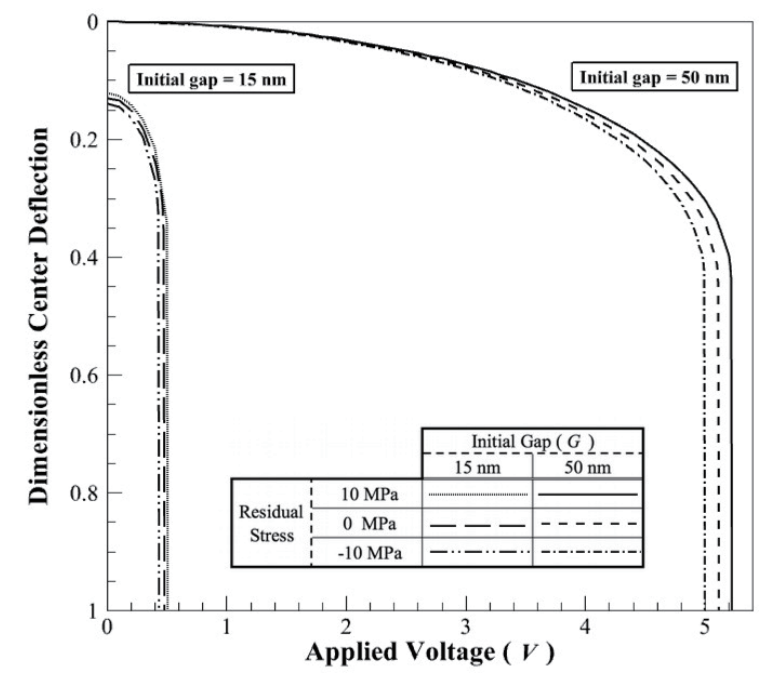

Fig. 4. Variation of dimensionless center-point deflection with applied voltage as a function of residual stress and initial gap size.

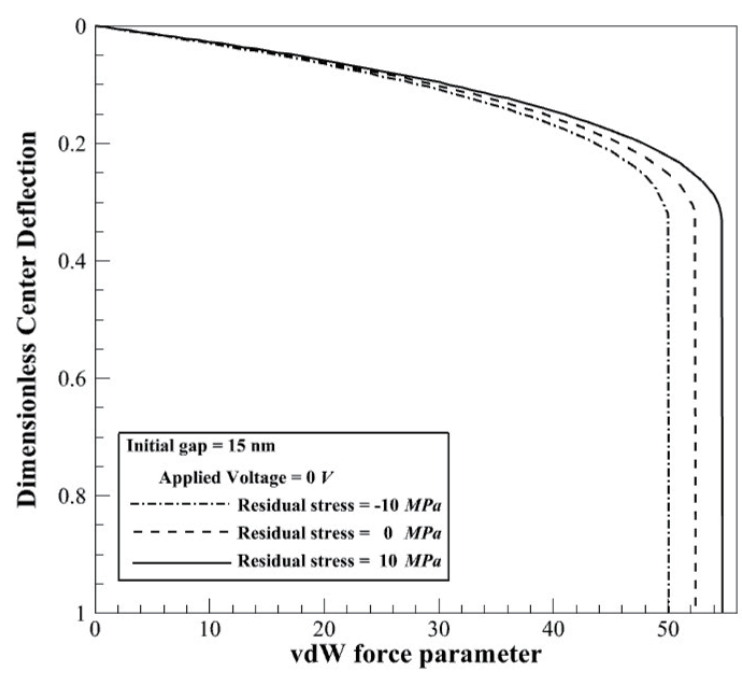

Fig. 6. Variation of dimensionless center-point deflection with van der Waals force parameter as a function of residual stress. (Note that no driving voltage is applied.)

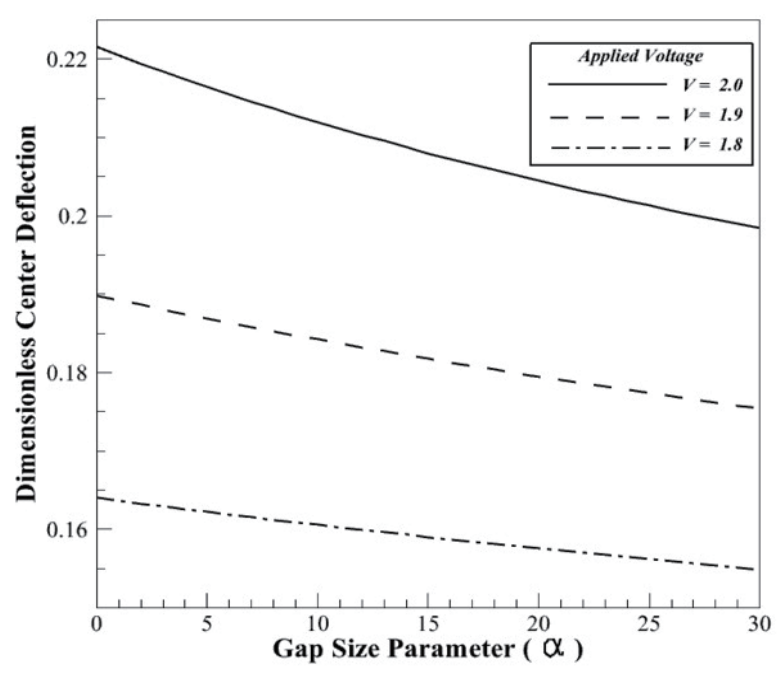

Fig. 5. Variation of dimensionless center-point deflection with gap size parameter as a function of applied voltage.

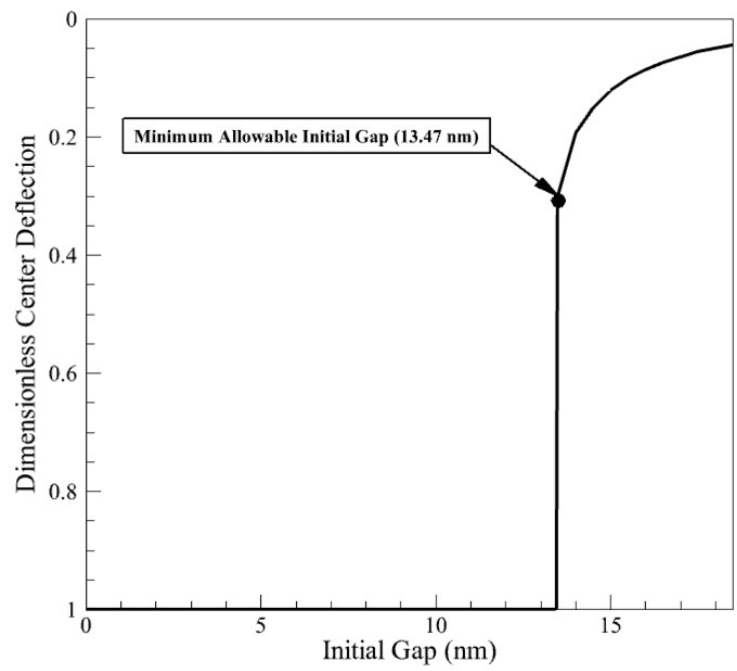

Fig. 7. Variation of dimensionless center-point displacement with initial gap size. (Note that no driving voltage is applied.)

Figure 6 shows the variation of the nanobeam center-point deflection with the van der Waals parameter as a function of residual stress. Note that the actuating voltage is set to zero such that beam deflection is a result of van der Waals forces alone. It is seen that for each value of the residual stress, the nanobeam deflection increases with increasing van der Waals parameter. Moreover, the pull-in phenomenon occurs when the van der Waals parameter reaches a certain critical value.

Figure 7 shows the variation of the nanobeam center-point deflection with the initial 
minimum gap size in the absence of an actuating force. Under this condition, the electrostatic force is set to zero and the variation of the dimensionless center-point displacement with the initial minimum gap is investigated. In this figure, with decreasing initial gap size between the two electrodes, surface traction is made through molecular interaction. The deflection of the nanobeam may be increased by the van der Waals force, and when the initial gap reaches its critical size (black point), the nanobeam suddenly collapses toward its substrate. Note that the infinity slope can also be observed in this figure. Therefore, the minimum allowable gap for an electrically actuated nanobeam can be determined using a hybrid numerical scheme.

\section{Conclusions}

In this study, we applied a hybrid numerical scheme comprising the differential transformation and FD approximation methods to analyze the nonlinear pull-in behavior and examine the minimum allowable gap of an electrically actuated double-clamped nanobeam. In contrast to previous studies, the governing equation developed in this study takes into account the fringing field effect, the axial residual stress effect, the geometric nonlinearity of midplane stretching, the Casimir force effect, the van der Waals force effect, and the residual stress effect. In general, the results have shown that the nanobeam becomes structurally unstable at driving voltages equal to or greater than the pull-in voltage and collapses and makes transient contact with the lower electrode as a result. In particular, the results have shown that the pull-in voltage decreases when the Casimir and van der Waals force effects are taken into account and when the stress state within the nanobeam changes from compressive to tensile. In a future study, the hybrid numerical scheme will be applied to investigate the dynamic response of more complex 2D NEMS-based nanostructures subject to combined DC/AC loading effects.

\section{Acknowledgments}

The authors gratefully acknowledge the financial support provided to this study by the Ministry of Science and Technology of Taiwan under Grant Number MOST 107-2221-E-018014 .

\section{References}

1 J. B. Ma, L. Jiang, and S. F. Asokanthan: Nat. Nanotechnol. 21 (2010) 505708.

2 S. Akita, Y. Nakayama, S. Mizooka, Y. Takano, T. Okawa, Y. Miyatake, and T. Nosaka: Appl. Phys. Lett. 79 (2001) 1691.

3 C. Stampfer, A. Jungen, R. Linderman, D. Obergfell, S. Roth, and C. Hierold: Nano Lett. 6 (2006) 1449.

4 E. S. Hung and S. D. Senturia: J. Microelectromech. Syst. 8 (1999) 497.

5 C. C. Liu and C. C. Wang: Appl. Math. Modell. 38 (2014) 3269.

6 H. Sadeghian, G. Rezazadeh, and P. M. Osterberg: J. Microelectromech. Syst. 16 (2007) 1334.

7 J. H. Kuang and C. J. Chen: Math. Comput. Modell. 41 (2005) 1479.

8 M. Tahani and A. R. Askari: Physica E 63 (2014) 151.

9 J. S. Duan and R. Rach: Int. J. Solids Struct. 50 (2013) 3511.

10 J. Yang, X. L. Jia, and S. Kitipornchai: J. Phys. D: Appl. Phys. 41 (2008) 035103.

11 A. Ramezani, A. Alasty, and J. Akbari: Int. J. Solids Struct. 44 (2007) 4925.

12 S. V. Rotkin: Electrochem. Soc. Proc. 6 (2002) 90. 
13 X. L. Jia, J. Yang, J. S. Kitipornchai, and C. W. Lim: Smart Mater. Struct. 19 (2010) 115028.

14 J. K. Zhou: Differential Transformation and Its Applications for Electrical Circuits (Huazhong University Press, Wuhan, 1986) (in Chinese).

15 Z. Šmarda, J. Diblík, and Y. Khan: Adv. Differ. Equations 1 (2013) 1.

16 C. C. Liu and C. Y. Lo: Int. Commun. Heat Mass Transfer 39 (2012) 1354.

17 C. K. Chen, B. S. Chen, and C. C. Liu: Int. Commun. Heat Mass Transfer 79 (2014) 750.

18 C. C. Liu: Smart Sci. 5 (2017) 132.

19 C. C. Liu: Microsyst. Technol. 23 (2017) 277.

20 C. K. Chen, H. Y. Lai, and C. C. Liu: Microsyst. Technol. 15 (2009) 813.

21 M. M. Zand and M. T. Ahmadian: Proc. Inst. Mech. Eng. C 224 (2010) 2037.

\section{About the Authors}
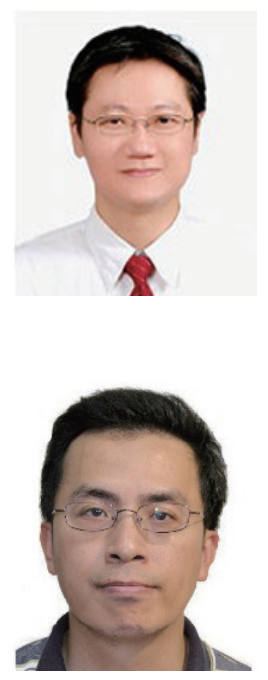

Cheng-Chi Wang received his B.S., M.A., and Ph.D. degrees in Mechanical Engineering from National Cheng Kung University, Taiwan in 1996, 1998, and 2001, respectively. His research interests include nonlinear dynamic analysis, nonlinear control, and numerical simulation. He now serves as a distinguished professor at the Graduate Institute of Precision Manufacturing of National Chin-Yi University of Technology. (wcc@ncut.edu.tw)

Chih-Jer Lin received his B.S., M.S., and Ph.D. degrees from National Cheng Kung University, Tainan, Taiwan, in 1992, 1994, and 1998, respectively, all in mechanical engineering. He is currently a professor at the Graduate Institute of Automation and Technology, National Taipei University of Technology, Taipei, Taiwan. His current research interests include mechatronics, vibration control, motion control, system identification, slidingmode control, robotics, and evolutionary algorithms. Professor Lin has been a guest editor of Applied Sciences and a member of the editorial board of the Journal of Chinese Society of Mechanical Engineers. (cjlin@ntut.edu.tw)

Chin-Chia Liu received his M.S. degree in Mechanical Engineering in 1999 and Ph.D. degree from National Cheng Kung University (NCKU) at Tainan in 2008. He is currently a professor at the Department of Industrial Education and Technology in National Changhua University of Education (NCUE), Taiwan. His current research interests include MEMS/NEMS dynamic behavior analysis, entropy generation analysis, and mechanical design. (ccliu@cc.ncue.edu.tw)

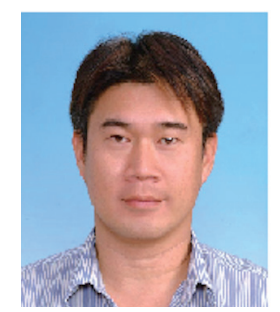

Chia-Chiang Hsu received his M.S. degree from National Chiayi University, Taiwan, in 2006. He was the District Chief of Shalu District in 2015-2017. He is currently the Chief, Sports Facilities Division Head of Taichung City Government Sports Bureau in Taiwan. Since 2016, he has been studying for a Ph.D degree at National Changhua University of Education. His research interests are in hydraulic engineering and numerical analysis. (hsu0309@yahoo.com.tw) 